\title{
Clinical and radiographically/neuroimaging documented outcome in transforaminal lumbar interbody fusion
}

\author{
John K. Houten, M.D., Nicholas H. Post, M.D., Joseph W. Dryer, M.D., \\ AND Thomas J. ERrico, M.D. \\ Department of Neurosurgery, Albert Einstein College of Medicine of Yeshiva University, Bronx; \\ and Departments of Neurosurgery and Orthopedic Surgery, New York University School of Medicine, \\ New York, New York
}

\begin{abstract}
Object. Although transforaminal lumbar interbody fusion (TLIF) is an increasingly popular surgical technique there are a limited number of studies in which investigators have stratified outcome data with respect to surgical indications or documented radiographically proven and clinical results with respect to disc space height (DSH). The authors conducted a study to evaluate the long-term outcomes after TLIF with respect to surgical indication and radiographic/neuroimaging results.

Methods. Thirty-three consecutive TLIF-treated patients underwent follow-up investigation for a mean of 37 months. Isthmic spondylolysis was present in eight patients, recurrent disc herniation in 14, and degenerative disc disease (DDD) in 11. The operative technique involved the placement of interbody structural allograft, pedicle screw instrumentation, and morcellized autograft in the anterior interbody space and the contralateral intertransverse gutter. Surgery was performed at L4-5 in 16 patients, L5-S1 in 14, L3-4 in two, and both L4-5 and L5-S1 in one patient.

Preoperative symptoms were back pain (in $91 \%$ of cases), leg pain (in 94\%), sensory loss (in 67\%), and motor deficits (in 30\%). Postoperatively, back pain was improved in $67 \%$ of the patients, unchanged in $27 \%$, and worsened in $7 \%$. Leg pain improved in $80 \%$ of patients, was unchanged in $10 \%$, and worsened in $10 \%$. Outcome, as measured using the Prolo Functional and Economic Scales, improved from a score of 4.9 to 7 . In patients with spondylolysis and recurrent disc herniation outcomes were better than in those with DDD only. There was no correlation of outcome with symptom duration, patient age, or level of surgery. In an independent review of pre- and late postoperative radiographs no significant differences in lordosis angles, Cobb angles, or DSHs were found. Fusion occurred in all cases.

Conclusions. The TLIF procedure was associated with good clinical outcomes and a high fusion rate but no change in the DSH. Patients who present with spondylolysis and recurrent herniations experience better outcome than those with degenerative disease alone.
\end{abstract}

\section{KEY WORDS - degenerative disc disease - spondylolisthesis - recurrent disc disease • transforaminal lumbar interbody fusion}

$\mathrm{T}$ RANSFORAMINAL lumbar interbody fusion is an increasingly popular technique that is used to achieve a circumferential arthrodesis in the lumbar spine. The TLIF procedure is similar to the more established PLIF in that it allows for an interbody fusion without necessitating a separate anterior approach. Compared with PLIF, however, TLIF offers several advantages. It involves the unilateral removal of a facet joint, and this more lateral approach to the disc space requires minimal retraction of the dura mater, thus reducing the potential for neurological injury. For the same reason, TLIF also can be performed at

Abbreviations used in this paper: $\mathrm{CT}=$ computerized tomography; DDD = degenerative disc disease; DSH = disc space height; $\mathrm{MR}=$ magnetic resonance; $\mathrm{PLIF}=$ posterior lumbar interbody fusion; TLIF $=$ transforaminal lumbar interbody fusion. the upper lumbar levels where proximity to the conus medullaris makes the dural tube retraction necessary in PLIF unfeasible. ${ }^{817}$ In addition, TLIF can be performed contralateral to the site of a prior laminotomy, thus avoiding adherent scar tissue.

The results reported on initial studies of TLIF have been promising; clinical outcomes and fusion rates appear comparable to those seen after PLIF or circumferential fusion performed via combined anterior-posterior approaches. ${ }^{11,15,17}$ There are still limited long-term follow-up data for TLIF, however, and few studies have included the stratification of outcome data with respect to surgical indications. The long-term effects of TLIF on DSHs, Cobb angles, and lordosis angles have also not been established, nor whether any of these measurements has any relationship with outcomes. 


\section{Clinical Material and Methods}

\section{Patient Selection and Follow Up}

During a 2-year interval between January 1998 and December 1999, 33 consecutive patients underwent TLIF performed by two spine surgeons (T.J.E. and J.W.D.) at a single institution, New York University School of Medicine. We conducted a retrospective review of office charts, hospital charts, and radiological studies to assess preoperative symptomatology, findings on clinical examination, and radiological/neuroimaging characteristics. The operating surgeons performed postoperative examinations during the course of routine follow-up office visits. Later followup examination was conducted by telephone interview. Clinical outcomes were assessed according to Prolo Functional and Economic Scale scores (Table 1). ${ }^{16}$

\section{Patient Population}

Of 33 patients, 19 were men (58\%) and 14 were women $(42 \%)$. The mean age at surgery was 42 years (range $25-58$ years). Twelve patients (36\%) were smokers and nine (27\%) were involved in Workers' Compensation proceedings. Twenty-two patients (67\%) had undergone prior lumbar surgery and five (15\%) had two or more of the following comorbidities requiring at least intermittent steroid agent use: coronary artery disease, diabetes, hypertension, and asthma.

Indications for surgery were isthmic spondylolysis with spondylolisthesis in eight patients (24\%), recurrent disc herniation in 14 (42\%), and DDD in 11 (33\%). An interbody fusion was recommended for patients with DDD only after the symptomatic level was confirmed by a positive response on discography. The mean duration of symptoms prior to surgery was 39 months (range 2-156 months). Long-term follow-up data were obtained in all patients (mean follow-up period 37 months, range 3-57 months).

Preoperative symptoms were back pain in $91 \%$ of cases, leg pain in $94 \%$, sensory loss in $67 \%$, and lower-extremity muscle weakness in 30\%. Surgery was performed at L4-5 in 16 patients, L5-S1 in 14, L3-4 in two, and both L4-5 and L5-S1 in one patient.

\section{Radiographic Assessment}

All available postoperative radiological/neuroimaging studies were reviewed independently for evidence of fusion by a neuroradiologist who was not involved in the patients' care and was unaware of clinical results. The criteria used to indicate fusion were as follows: 1 ) the absence of movement on flexion-extension x-ray films; 2) presence of bone bridging between the graft and adjacent vertebral endplates; and 3) lack of lucency around spinal instrumentation. Preoperative and postoperative lordosis angles, Cobb angles, and DSHs were measured. ${ }^{2,18}$ The anterior, middle, and posterior DSHs were measured on lateral radiographs, and the midpoint in the anterior-posterior dimension and points one third and two thirds from the front were recorded. ${ }^{18}$

\section{Surgical Technique}

With the patient in the prone position, a subperiosteal dissection of the posterior elements, including the trans-
TABLE 1

Summary of the Prolo Functional and Economic Scales

\begin{tabular}{cl}
\hline \hline \multicolumn{1}{c}{ Score } & \multicolumn{1}{c}{ Criteria } \\
\hline $\begin{array}{c}\text { functional status } \\
1 \\
2\end{array}$ & $\begin{array}{l}\text { total incapacity } \\
\text { mild to moderate level of low-back pain \&/or } \\
\text { sciatica } \\
\text { low level of pain \& able to do everything except } \\
\text { sports } \\
4\end{array}$ \\
& no pain, but has had $>1$ recurrence of low-back \\
& pain or sciatica \\
5 & complete recovery w/o recurrent pain, no activi- \\
& ty restriction \\
1 & complete disability \\
2 & no gainful occupation but can do housework or \\
3 & some retirement activities \\
4 & able to work but not at previous occupation \\
& able to work at previous occupation but w/ re- \\
5 & strictions or limited status \\
& able to perform previous occupation w/o restric- \\
& tions
\end{tabular}

verse processes, was performed via a midline incision. Material for a corticocancellous autograft to be used later in the procedure was then harvested through a separate facial incision. In patients with radiculopathy, a unilateral laminotomy and a facetectomy were performed, always on the side ipsilateral to the lower-extremity symptoms. After insertion of transpedicular K-wires, pedicle screws were placed using external anatomical landmarks and intraoperative x-ray films as guides. On the side of the laminotomy, the pedicles were felt from within the canal to verify that no cortical breach was present. Computerized image guidance, fluoroscopy, and electrophysiological monitoring were not used.

The exiting nerve root was identified and protected with a small dissector while a discectomy was performed on the thecal sac with minimal medial retraction. Osteotomes were used to remove the osseous posterior lip of the vertebra and thus provide a parallel channel that allowed acceptance of the interbody graft. With the aid of gentle distraction against the pedicle screws to increase the DSH, large looped curettes were used to remove any remaining cartilaginous endplate within the disc space, although care was taken not to perforate the anterior annulus.

Once the discetomy was complete, morcellized autograft was packed into the anterior half of the disc space. Posterior to the autograft, either a semilumar piece of femoral allograft or two fibular cross-sectional pieces of allograft were positioned to fit snugly into the disc space. The posterior instrumentation was then tightened under modest compression to allow placement of the interbody allograft. The intertransverse gutter contralateral to the laminotomy was then decorticated and packed with additional autograft material. The side of the facetectomy was not usually packed with any graft material.

\section{Statistical Analysis}

Statistical data were analyzed using Prism 4 software (GraphPad Software, Inc., San Diego, CA). Descriptive statistics were calculated for patient demographics. The Stu- 
dent t-test was used to assess differences in DSHs, Cobb angles, and lordosis angles. Possible changes in pre- and postoperative Prolo Scale scores were assessed using the Wilcoxon signed-rank test. Intergroup comparisons were analyzed with the Mann-Whitney U-test. A probability value less than 0.05 was considered significant.

\section{Results}

We found subjective improvement in symptoms of back pain in $20(67 \%)$ of the 30 patients suffering from this symptom preoperatively, whereas symptoms were unchanged in eight patients $(27 \%)$ and worse in two patients $(7 \%)$. Of the 31 patients with preoperative leg pain, symptoms improved in $25(80 \%)$, were unchanged in three (10\%), and became worse in three (10\%). Preoperative lower-extremity sensory loss in 22 patients improved after surgery in 17 $(77 \%)$, remained the same in one $(5 \%)$, and became worse in four $(18 \%)$. Preoperative lower-extremity muscle weakness in 10 patients improved postoperatively in five $(50 \%)$, was unchanged in four (40\%), and worsened in one $(10 \%)$. A summary of the patient symptomatology is presented in Table 2.

After surgery, there was a significant improvement in the mean Prolo Scale score from 4.9 to 7.0 ( $p<0.0001)$. Based on their surgical indications, patients were divided into three groups: spondylolysis, recurrent disc herniation, and DDD. In the groups with spondylolysis and recurrent disc herniation there was significant improvement in Prolo Scale score ( $\mathrm{p}<0.023$ and $\mathrm{p}<0.001$, respectively). The improvement in the DDD group Prolo Scale score was not significant, however (Table 3 ).

There was no correlation among patient age, preoperative symptom duration, or spinal level with Prolo Scale score outcomes. Workers' Compensation proceedings were ongoing in nine patients, but there was no difference in their outcomes compared with that in patients not receiving such compensation. There was a trend that neared statistical significance: the Prolo Scale scores improved less in the five patients with two or more medical comorbidities compared with those with one or no medical comorbidities $(\mathrm{p}<0.052)$.

No intraoperative complications or new postoperative motor deficits were observed. One patient, however, complained of severe lower-extremity radicular pain starting immediately after surgery. Subsequent CT and MR imaging revealed satisfactory instrumentation placement and no evidence of nerve root impingement. The pain sub-

TABLE 2

Preoperative symptoms and change after TLIF in 33 patients*

\begin{tabular}{llllr}
\hline \hline \multirow{2}{*}{ Preop Symptom } & \multicolumn{4}{c}{ No. of Patients (\%) } \\
\cline { 2 - 5 } & Improved & Same & Worse & Total Overall \\
\hline back pain & $20(67)$ & $8(27)$ & $2(7)$ & $30(91)$ \\
leg pain & $25(80)$ & $3(10)$ & $3(10)$ & $31(94)$ \\
LE sensory loss & $17(77)$ & $1(5)$ & $4(18)$ & $22(67)$ \\
LE motor deficit & $10(30)$ & $5(50)$ & $4(40)$ & $1(10)$ \\
\hline
\end{tabular}

$*$ LE $=$ lower-extremity.
TABLE 3

Summary of Prolo Scale scores stratified by surgical indication*

\begin{tabular}{lccc}
\hline \hline & \multicolumn{3}{c}{ Mean Score } \\
\cline { 2 - 4 } Score Category & $\begin{array}{c}\text { Isthmic } \\
\text { Spondylolysis } \dagger\end{array}$ & $\begin{array}{c}\text { Recurrent } \\
\text { Disc Herniation } \dagger\end{array}$ & DDD \\
\hline preop & & & \\
functional & 2.0 & 2.1 & 2.3 \\
economic & 3.0 & 3.1 & 2.4 \\
combined & 5.0 & 5.2 & 4.7 \\
postop & & 3.9 & 2.7 \\
functional & 3.9 & 4.1 & 2.9 \\
economic & 4.4 & 8.0 & 5.6 \\
combined & 8.3 & & \\
\hline
\end{tabular}

* There were eight patients with spondylolysis, 14 with recurrent disc herniation, and 11 with DDD.

$\dagger$ Statistically significant improvement was found in patients with spondylolysis $(\mathrm{p}<0.023)$ and recurrent disc herniation $(\mathrm{p}<0.001)$.

sided spontaneously after several weeks. A second patient had a superficial wound infection that was effectively treated with a course of oral antibiotic medication.

During the course of routine office follow up, postoperative $\mathrm{x}$-ray films obtained in all patients were reviewed by the operating surgeons, and fusion was judged to have occurred in all cases. Preoperative and postoperative radiographs acquired at a mean of 11 months after surgery (range 6-22 months) were available for independent review in 23 cases, and the criteria for solid fusion were met in all of these. No difference was found when comparing pre- and the final postoperative $\mathrm{x}$-ray films with respect to lordosis angles, Cobb angles, or DSHs. The anterior, middle, and posterior DSH measurements did not change during the course of sequential postoperative studies (Figs. 1 and 2).

Review of the last postoperative $\mathrm{x}$-ray films revealed no evidence of significant disease at adjacent levels. Three patients underwent hardware removal after the fusion mass had consolidated, but no patient underwent any other lumbar surgery during the follow-up period.

\section{Discussion}

Our experience with the TLIF procedure confirms the findings of prior studies in that it produces good clinical outcomes, high rates of successful arthrodesis, and a very low incidence of complications. It provides circumferential fusion via a posterior approach and, thus, avoids the need for a separate anterior surgery that would entail additional risk of retrograde ejaculation or injury to abdominal viscera or vascular structures. In addition, TLIF avoids the need for dural retraction present when performing a PLIF, which may increase the potential for complications such as neurapraxic injury and dural laceration.

In patients with DDD that was visible on studies, a statistically significant improvement in Prolo Scale scores was not observed. The number of patients in this group is far too small to allow us to comment on the efficacy of interbody fusion in patients in whom discography had revealed DDD. There have been, however conflicting results reported in the literature regarding the optimal treatment of these patients. ${ }^{9,13,14}$ Our finding that spondylolysis 

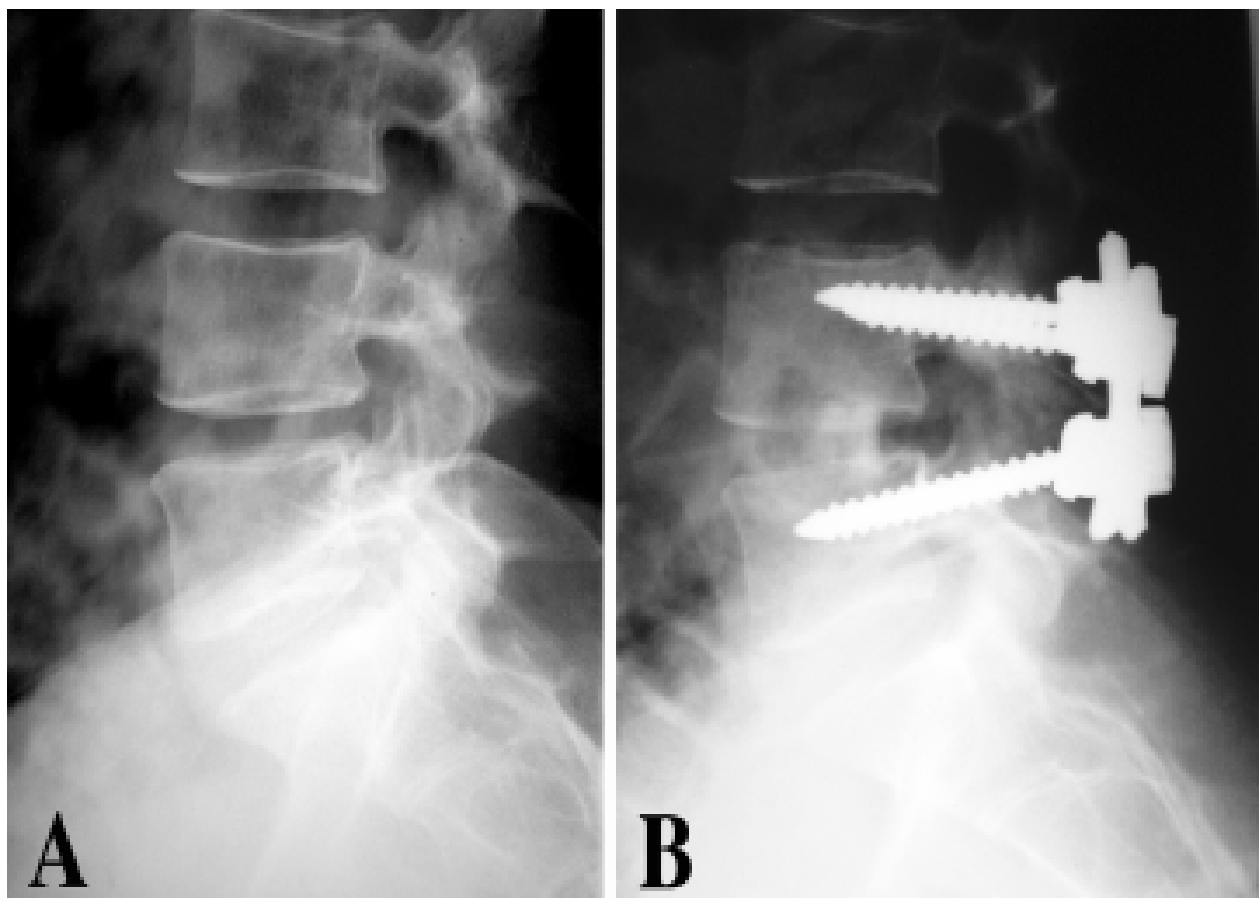

FIG. 1. Lateral lumbar radiographs obtained before (A) and 6 months after (B) an L4-5 TLIF for recurrent disc herniation.

and recurrent disc herniation were associated with better outcomes might be valuable in setting realistic expectations when the TLIF procedure is discussed with a patient.

Distraction of a lumbar disc space serves to increase the cross-sectional area of the neural foramen and has been assumed to be of clinical value in relieving neural compression. ${ }^{6}$ Although the importance of restoration of DSH and segmental lordosis has been emphasized in numerous works, there are limited experimental data to validate these concepts in clinical practice. ${ }^{4,5,12}$ In many recent investigations, the researchers were unable to correlate these parameters with patient satisfaction in individuals who underwent lumbar interbody fusions. ${ }^{3,7,19,20}$ Cheung, et al., ${ }^{7}$ have noted in their clinical series of lumbar fusions that, despite achieving an initial distraction of the DSH, there was a
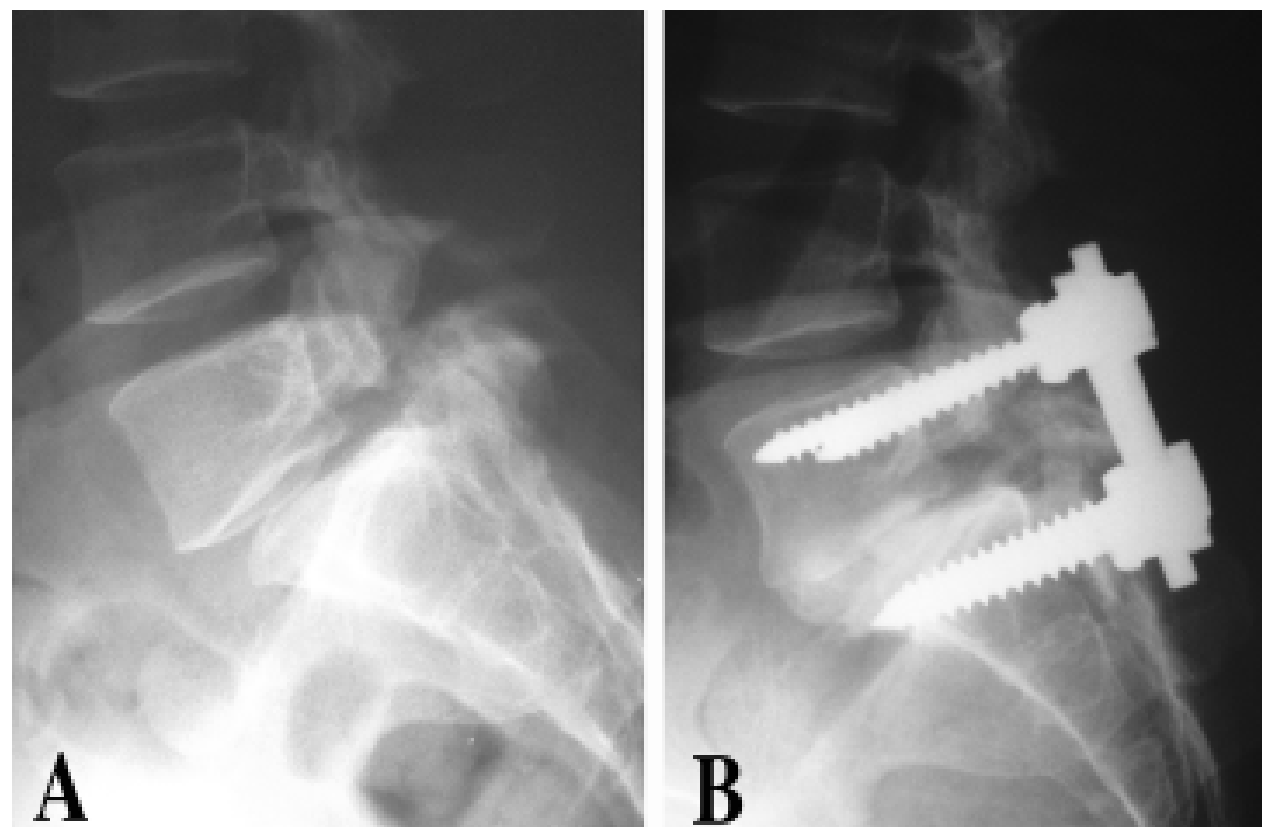

FIG. 2. Lateral lumbar radiographs acquired before (A) and 11 months after (B) an L5-S1 TLIF for spondylolysis with spondylolisthesis. No change was found in DSH. 
strong tendency for the disc space to collapse back into the preoperative position despite the presence of instrumentation. They speculated, therefore, that because the favorable clinical outcomes did not change following relapse back to the preoperative disc space height, disc space distraction might not be an important factor. ${ }^{7}$

In a recent study of pedicle screw-instrumented fusion surgery for spondylolysis investigators compared interbody fusion combined with disc space distraction to posterolateral fusion alone. ${ }^{10}$ The addition of interbody fusion produced a construct with greater mechanical strength but did not result in improved clinical outcomes. These findings have stimulated critical analysis of the role of disc space distraction in lumbar fusion procedures. ${ }^{1}$

In our study we demonstrated excellent clinical outcomes without any significant changes in DSH. A possible explanation for this might be that the elimination of segmental motion could stop irritation of a nerve root and result in symptomatic improvement without altering the dimensions of the neural foramen.

A contrary consideration, however, is that a laminotomy and a facetectomy, while allowing decompression of the posterior aspect of the exiting nerve root, do not increase the space between the pedicle boundaries of the foramen. It is possible that our failure to increase this dimension could have led to this phenomenon in the three patients with worse leg pain after surgery.

\section{Conclusions}

In cases in which a clinician has determined that a circumferential fusion is indicated, our results confirm that TLIF is an option that is associated with good clinical outcomes, a low morbidity rate, and high fusion rate. Our technique did not involve aggressive distraction of the disc space, and no difference was found between pre- and postoperative DSHs, Cobb angles, or lordosis angles in our patients. No relationship was detected between outcomes and change in the DSHs, Cobb angles, or lordosis angles, suggesting that aggressive disc space distraction, with its accompanying risk of neurapraxic injury, is not necessary. The clinical outcomes in patients with spondylolysis and recurrent disc herniation are better than those for patients with symptoms due to DDD alone. This latter finding serves to emphasize the necessity of careful patient selection to achieve excellent clinical results.

\section{References}

1. Benzel EC: Disc interspace distraction: is it really necessary? J Neurosurg 99 (2 Suppl):141-142, 2003

2. Bernhardt M, Bridwell KH: Segmental analysis of the sagittal plane alignment of the normal thoracic and lumbar spines and thoracolumbar junction. Spine 14:717-721, 1989

3. Beutler WJ, Peppelman WC Jr: Anterior lumbar fusion with paired BAK standard and paired BAK Proximity cages: subsidence incidence, subsidence factors, and clinical outcome. Spine J 3:289-293, 2003
4. Brantigan JW, Neidre A: Achievement of normal sagittal plane alignment using a wedged carbon fiber reinforced polymer fusion cage in treatment of spondylolisthesis. Spine J 3: 186-196, 2003

5. Burkus JK: Intervertebral fixation: clinical results with anterior cages. Orthop Clin North Am 33:349-357, 2002

6. Chen D, Fay LA, Lok J, et al: Increasing neuroforaminal volume by anterior interbody distraction in degenerative lumbar spine. Spine 20:74-79, 1995

7. Cheung KM, Zhang YG, Lu DS, et al: Reduction of disc space distraction after anterior lumbar interbody fusion with autologous iliac crest graft. Spine 28:1385-1389, 2003

8. Humphreys SC, Hodges SD, Patwardhan AG, et al: Comparison of posterior and transforaminal approaches to lumbar interbody fusion. Spine 26:567-571, 2001

9. Knox BD, Chapman TM: Anterior lumbar interbody fusion for discogram concordant pain. J Spinal Disord 6:242-244, 1993

10. La Rosa G, Conti A, Cacciola F, et al: Pedicle screw fixation for isthmic spondylolisthesis: does posterior lumbar interbody fusion improve outcome over posterolateral fusion? J Neurosurg 99 (2 Suppl): 143-150, 2003

11. Lowe TG, Tahernia AD: Unilateral transforaminal posterior lumbar interbody fusion. Clin Orthop Relat Res 394:64-72, 2002

12. Luk KD, Chow DH, Holmes A: Vertical instability in spondylolisthesis: a traction radiographic assessment technique and the principle of management. Spine 28:819-827, 2003

13. Madan S, Gundanna M, Harley JM, et al: Does provocative discography screening of discogenic back pain improve surgical outcome? J Spinal Disord Tech 15:245-251, 2002

14. Madan SS, Harley JM, Boeree NR: Circumferential and posterolateral fusion for lumbar disc disease. Clin Orthop Relat Res 409:114-123, 2003

15. Moskowitz A: Transforaminal lumbar interbody fusion. Orthop Clin North Am 33:359-366, 2002

16. Prolo DJ, Oklund SA, Butcher M: Toward uniformity in evaluating results of lumbar spine operations. A paradigm applied to posterior lumbar interbody fusions. Spine 11:601-606, 1986

17. Salehi SA, Tawk R, Ganju A, et al: Transforaminal lumbar interbody fusion: surgical technique and results in 24 patients. Neurosurgery 54:368-374, 2004

18. Saraste H, Brostrom LA, Aparisi T, et al: Radiographic measurement of the lumbar spine. A clinical and experimental study in man. Spine 10:236-241, 1985

19. Schiffman M, Brau SA, Henderson R, et al: Bilateral implantation of low-profile interbody fusion cages: subsidence, lordosis, and fusion analysis. Spine J 3:377-387, 2003

20. Soini J: Lumbar disc space heights after external fixation and anterior interbody fusion: a prospective 2-year follow-up of clinical and radiographic results. J Spinal Disord 7:487-494, 1994

\footnotetext{
Manuscript received December 8, 2005.

Accepted in final form January 31, 2006.
}

Address reprint requests to: Nicholas H. Post, M.D., Department of Neurosurgery, New York University School of Medicine, New Bellevue 7 South 4, 462 First Avenue, New York, New York 10016. email: postn01@med.nyu.edu. 